\title{
EMERGENT SELF-SIMILARITY AND SCALING PROPERTIES OF FRACTAL INTRA-URBAN HEAT ISLETS FOR DIVERSE GLOBAL CITIES
}

\author{
A PREPRINT \\ Anamika Shreevastava ${ }^{1, *}$, P. Suresh C. Rao ${ }^{1}$, Gavan S. McGrath ${ }^{2,3}$ \\ 1. Lyles School of Civil Engineering, Purdue University, West Lafayette, IN, USA \\ 2. School of Earth and Environment, The University of Western Australia, Perth, Australia \\ 3. Ecosystem Science, Department of Biodiversity Conservation and Attractions, Kensington, WA, Australia \\ *Corresponding author: ashreeva@purdue.edu; http://anamika255.github.io
}

September 11, 2019

\begin{abstract}
Urban areas experience elevated temperatures due to the Urban Heat Island (UHI) effect. However, temperatures within cities vary considerably and their spatial heterogeneity is not well characterized. Here, we use Land Surface Temperature (LST) of 78 global cities to show that the Surface UHI (SUHI) is fractal. We use percentile-based thermal thresholds to identify heat clusters emerging within SUHI and refer to them collectively as intra-urban heat islets. The islets display properties analogous to that of a percolating system as we vary the thermal thresholds. At percolation threshold, the size distribution of these islets in all cities follows a power-law, with a scaling exponent $(\beta)$ of $1.88( \pm 0.23,95 \% C I)$ and an aggregated Perimeter Fractal Dimension $(D)$ of 1.33 $( \pm 0.064,95 \% C I)$. This commonality indicates that despite the diversity in urban form and function across the world, the urban temperature patterns are different realizations with the same aggregated statistical properties. Furthermore, we observe the convergence of these scaling exponents as the city sizes increase. Therefore, while the effect of diverse urban morphologies is evident in smaller cities, in the mean, the larger cities are alike. Lastly, we calculate the mean islet intensities, i.e. the difference between mean islet temperature and thermal threshold, and show that it follows an exponential distribution, with rate parameter, $\lambda$, for all cities. $\lambda$ varied widely across the cities and can be used to quantify the spatial heterogeneity within SUHIs. In conclusion, we present a basis for a unified characterization of urban heat from the spatial scales of an urban block to a megalopolis.
\end{abstract}

\section{Introduction}

Cities are the apex examples of complex, coupled, socio-technological systems, which are projected to account for more than $70 \%$ of the global population by 2050 (Seto and Shepherd, 2009). Rapid urbanization presents multiple challenges, among them the Urban Heat Island (UHI) effects. Urban heat stress is predicted to be more frequent 
and persistent in the coming century due to a synergistic effect of mesoscale heat waves and the UHI (Li and BouZeid, 2013; Meehl and Tebaldi, 2004; Allen et al., 2014). Metrics such as UHI Intensity, that quantify the difference between a representative (often the mean) urban and neighboring non-urban air temperature, fail to characterize intraurban spatial variability (Debbage and Shepherd, 2015; Stewart and Oke, 2012). Furthermore, critical hot regions can emerge within the heat island itself. Therefore, for optimizing mitigation efforts and targeting scarce resources where they are most warranted, it is critical to characterize the spatial heterogeneity that arises within a city (Rosenzweig et al., 2010).

Cities tend to be warmer because of an increase in heat sources, such as excessive built-up area, industries, and airconditioning exhausts, and a scarcity of heat sinks (e.g., vegetation and water bodies) (Oke, 1982). Spatial organization of physical assets, i.e., the urban form (e.g., impervious areas; buildings), as well as mobile assets such as automobile govern the distribution of heat sources in a city and modify the cooling effect of heat sinks. Prior research has shown that urban form has numerous fractal properties related to land use (Batty and Longley, 1994), urban infrastructure networks (Yang et al., 2017; Krueger et al., 2017), and impervious area (Chen, 2010; Makse et al., 1998). Similarly, the metabolic functions of cities (Oke, 1982) display scaling in the spatial patterns of population distribution, traffic, and energy use among others (Gonzalez et al., 2008; Rozenfeld et al., 2008; Bettencourt and West, 2010). While similar scaling laws and fractal metrics have also been developed in atmospheric sciences (Lovejoy and Schertzer, 1986), their application in UHI studies remains limited (Weng, 2003; Debbage and Shepherd, 2015). Comprehensive scaling laws that describe spatio-temporal variability of intra-urban high heat clusters have not been explored yet.

Based on the established correlation of surface temperatures and urban morphology (Buyantuyev and Wu, 2010; Zhou et al., 2011; Liu and Weng, 2009), we hypothesize that SUHI patterns should exhibit a fractal spatial structure. We analyze Landsat 8 derived LST data for 78 diverse cities across the world and use percentile-based thermal thresholds and clustering techniques from percolation theory to identify clusters of high heat within cities. Here, we refer to the collection of heat clusters as intra-urban heat islets, which combine to form the UHI as a whole. First, we demonstrate the statistical self-similarity of heat islets. We then identify the scaling laws that quantify their size and intensity distributions, thereby, developing new metrics for spatial characterization of SUHIs.

\section{Methodology}

\subsection{Data}

We initially sampled a wide variety of global cities, including but not limited to the C-40 (http://www.c40.org/cities), that are representative of diverse climate types (Peel et al., 2007) as well as cultural backgrounds. Since the focus of this study is intra-urban heat islets, only the cities that exhibited elevated temperatures within the urban boundaries were selected. Cities which showed inversion of the heat island effect (Lazzarini et al., 2013) or contained significant topographic relief dominating the LST patterns were removed from the sample. The resulting sample set consists of 78 cities with populations ranging from 200k to 30M. It includes densely packed urban areas, such as Seoul and Beijing, agglomerated cities such as Mexico City, highly heterogeneous cities like Mumbai, and highly structured, grid-like cities such as Los Angeles and Houston. It should be noted that the selected list is not exhaustive in any way but a representative subset of diverse global cities. Complete list of cities studied and their Landsat image used is attached as Dataset S1 of Supplementary Material.

For obtaining spatially rich datasets for intra-urban studies, satellite-based observations have proven increasingly useful. Remotely sensed Land Surface Temperature (LST) is used as an indicator to characterize the Surface Urban Heat Island (SUHI) (Voogt and Oke, 2003). Furthermore, uniformity in data quality of remotely sensed observations enables multi-city comparisons (Imhoff et al., 2010; Peng et al., 2011; Zhou et al., 2013). The geospatial analysis was implemented using Google Earth Engine (GEE) (Gorelick et al., 2017) to filter out cloud-free summertime days with an incident solar angle of at least 60 degrees for the selected cities. Figure 2 serves to visualize the geospatial format of 
data collected using the example of Boston, USA, and Kolkata, India. Land Surface Temperature (LST) was derived by a Single Channel Algorithm as detailed in (Walawender et al., 2012) using data from Landsat 8 (Bands 4, 5, 10, and 11) daytime images at a resolution of $90 \mathrm{~m}$ (Figs 2b, and 2f). See Appendix A for algorithm and Dataset S1 further information on Landsat scenes used. For each city, the urban area was estimated using Land Cover Type dataset of Moderate-resolution Imaging Spectroradiometer (MODIS) - MCD12Q1 (Figs 2a, and 2e). The exact definition of urban boundaries and city area plays a significant role in urban scaling laws where different urban extents can produce different scaling exponents (Cottineau et al., 2017), therefore, a buffer of $5 \mathrm{~km}$ in the rural regions was taken to account for the peri-urban settlements. However, as the heat islets occur well within the city boundaries, the scaling exponents were found to be independent of the buffer width. Lastly, in case of coastal cities, the Large Scale International Boundary (LSIB) dataset provided by United States Office of the Geographer was used to crop out the oceans and delineate coastal boundaries within the GEE environment.

\subsection{Heat Islets clustering and fractal analysis}

We conceptualize the thermal map as a Digital Elevation Model (DEM) where temperatures substitute for elevation (See figure 7 in Appendix B). For each city, we select regions with temperatures above specified percentile thresholds $\left(T_{t h r}\right)$ and group the connected regions together using a Moore neighborhood to define clusters, thereby identifying islets of higher heat for each incremental threshold (Shreevastava et al., 2018). In figure 2, we use the example of Boston and Kolkata to demonstrate the collection of islets appear at two different thermal thresholds, one corresponding to the percolation threshold, and another corresponding to the $95^{\text {th }}$ percentile. At higher temperature thresholds we can delineate areas within cities that experience extreme temperatures. The use of thermal percentiles enables comparison between cities which differ in their background climates as apparent in figures $2 b$ and $2 f$ where the range of temperatures vary significantly between the two cities. We utilize two metrics to characterize the spatial complexity of these islets, as described below.

As a primary test of fractal structure, the aggregated Area-Perimeter fractal dimension (Mandelbrot, 1975) of the collection of islets is estimated at each $T_{t h r}$ using the following equation:

$$
\Sigma P=k \cdot \Sigma A^{\frac{D}{2}}
$$

where $D$ is the fractal dimension, $k=2 * \sqrt{\pi}=3.545$, that is determined for the limiting case of a circle, and the summation of perimeters $(P)$ and areas $(A)$ goes over the set of islets (Batty and Longley, 1994). Note that we are referring to the fractal dimension of the ensemble iso-thermal contour lines here. In the limiting case of a circle, $P \propto \sqrt{ } A$ and $D=1$. For more irregular and convoluted shapes, the perimeter becomes increasingly plane-filling or elongated, resulting in the limit in linear shapes where $P=A$ and $D=2$ (Figure $4 \mathrm{a}$ and $4 \mathrm{~b}$ ). For statistically self-similar surfaces, not only is D a fractional value between 1 and 2, but it is also the same for all thresholds used for clustering (Isichenko and Kalda, 1991).

Second, we examine the size distribution of islets. As $T_{t h r}$ is decreased, the total number of clusters increase as more regions with $T>T_{t h r}$ are selected. However, at the percolation threshold, the number of clusters start declining as they coalesce to form a giant connected component. This is illustrated in figure 3 using the example of Boston, USA. For fractal landscapes, clusters are statistically self-similar at the percolation threshold over certain ranges of sizes, with the cluster areas following a probability distribution with a power-law tail (Isichenko and Kalda, 1991). This was first presented as an empirical rule by physicist and geographer Korcak (Imre and Novotnỳ, 2016), who suggested a general scaling law, now referred to as the Korcak's law or the number-area rule, describing the sizedistribution of various geographical objects, including lakes and islands (Mandelbrot, 1975; Cael and Seekell, 2016). This is expressed as the relative number of islands with an area equal to a is given by the power-law: $N(a) \propto a^{-\beta}$. As an exceedance probability distribution function, the size distribution can be written as the following 


$$
P(A \geq a) \propto a^{1-\beta}, \quad \forall a \geq a_{\min }
$$

Above the percolation threshold, deviations from the power-law result in some form of tempering. We used a conservative approach to test for and fit the power-law distributions using a combination of maximum-likelihood fitting methods with goodness-of-fit tests based on the Kolmogorov Smirnov (KS) statistic and likelihood ratios (Clauset et al., 2009) (See Appendix C for detailed methodology).

\section{Results and Discussions}

\subsection{Fractal Dimension}

The aggregated area-perimeter Fractal Dimension $(D)$ of the heat islets was calculated for multiple values of $T_{t h r}$ $\left(50^{\text {th }}, 60^{\text {th }}, \cdots, 90^{\text {th }}\right.$ percentiles $)$. For each city, $D$ is consistent for all values of $T_{t h r}$ as shown by the same $\log$ (Area) $\log ($ Perimeter) ratio (Figure $4 \mathrm{a}$ and $4 \mathrm{~b}$ ). This is a key finding, demonstrating the statistical self-similarity within SUHIs, empirically establishing fractal geometry of urban thermal landscape. Furthermore, the calculated values of $D$ across all cities were approximately normally distributed with a mean $D=1.33$ and standard deviation (s.d.) of 0.033. (see Figures 4c and 4d, see Dataset S3 of Supplementary Material for a complete list). Makse et al., (1998)(Makse et al., 1998) reported $1.2<D<1.4$ for clusters of urban impervious areas, with a mean value of 1.33 as well. Another study reported $1.22 \pm 0.08$ for 68 Chinese cities (Chen, 2010). Therefore, the fractal dimensions of SUHI are in agreement with that of urban impervious area.

$D$ scaled weakly with city size as $D=0.0695 \cdot \log A_{\text {city }}+1.15\left(R^{2}=0.7\right)$ (Figure 4 d). The tendency for $D$ to be smaller for small cities is reflective the varying urban morphology of cities as they grow. Smaller cities are often mono-centric (more circle-like) with fewer heat islets, as a result, we would expect $D$ to tend toward a value of 1 . While megalopolises, on the other hand, formed from agglomeration of multiple peri-urban settlements are expected to have higher number of heat islets scattered throughout the city, thereby, increasing $D$ (Figure 4d). This is also reflected in the total number of islets for each city that scales linearly as $N=0.038 * A_{\text {city }}+40\left(R^{2}=0.8\right)$ (See figure 8 in Appendix B). However, for self-affine surfaces, the total perimeter is dominated by the smallest islets, and the total area is dominated by the largest island (Isichenko and Kalda, 1991). To examine the average shape of an islet within a city, area-weighted mean fractal dimension (AWMFD) of the islets is a useful alternative (Debbage and Shepherd, 2015). It is calculated using the following equation:

$$
A W M F D=\sum_{i=1}^{n}\left[\left(\frac{2 \ln \left(\frac{p_{i}}{k}\right)}{\ln a_{i}}\right)\left(\frac{a_{i}}{\sum_{i=1}^{n} a_{i}}\right)\right]
$$

The AWMFD for cities were found to be approximately normally distributed as well with a mean AWMFD $=1.227$ (s.d. = 0.025; See figure 9 in Appendix B).

\subsection{Islet Size distribution}

At the percolation threshold, the area-exceedance probability distribution was found to scale consistently with a powerlaw tail for all cities, with the scaling exponent normally distributed with mean $\beta=1.88$ and s.d. $=0.12$ (Figures 5a and 5b). Alternative distributions, such as log-normal, exponential and Weibull, were tested as potential candidates; however, they were all rejected (at $p>0.1$ ), while the same tests suggested that the distributions could not be rejected as having power-law tails (See Appendix C and Dataset S2 of Supplementary Material). 
The power-law size distribution is another key finding that further supports the observed fractal structure of heat islets. The percolation threshold was found to be closely associated with the statistical mode of temperature distribution, i.e. the most frequently encountered temperature in the city $\left(R^{2}=0.93\right.$, see figure 10 in Appendix B). For the case uncorrelated percolation, $\beta$ is estimated to be $187 / 91$ ( 2.05) (Isichenko, 1992; Sahimi and Sahimi, 2014). Moreover, empirical distributions of land classified as urban and cities modeled with correlated percolation as well have found similar size distributions with $\beta \sim 2$ (Makse et al., 1998; Fluschnik et al., 2016; Gangopadhyay and Basu, 2009). A slightly smaller exponent of 1.88 , in this case, indicates a greater probability of occurrence of heat islets than what would be expected from impervious area alone. Here, the power-law tails are curtailed on the higher end by limits of the study domain i.e. the total city size, in this case, (Newman, 2005), and on the lower end, by spatial resolution. Numerous smaller heat clusters are either not captured or are rounded off to integer multiples of the lowest available resolution. Interestingly, in this case, the lower bound $\left(a_{\min }\right.$ at which the power-law tail starts) is $\sim 0.25$ $\mathrm{km}^{2}$, which corresponds to the size of a couple of urban blocks. This suggests that below this, the heat islets may indeed scale differently as the individual building level features become evident. There is potential to extend this analysis beyond these spatial scales, however, this was not the objective of this study. A relationship between $D$ and $\beta$ can be derived for Gaussian surfaces as $\beta-1=D / 2$ (Isichenko and Kalda, 1991). However, this was not found to be true for heat islets indicating a departure from random Gaussian topography. Lastly, fractal landscapes are expected to yield the same scaling exponents irrespective of the resolution. To test their sensitivity to input resolution, LST maps were aggregated at a range of resolutions from $90 \mathrm{~m}$ to $720 \mathrm{~m}$. Scaling exponents were found to be the same, adding further support to the self-similar topography of SUHI.

At temperatures above the percolation threshold, the size distribution shows a deviation from power-law in the form of exponential tempering (Clauset et al., 2009) suggesting a model more consistent with:

$$
P(A \geq a) \propto a^{1-\beta} \cdot e^{-c\left(T_{t h r}\right) \cdot a}, \quad \forall a \geq a_{\min }
$$

where $c$ is a tempering coefficient that depends on the thermal threshold. As the $T_{t h r}$ moves further away from the percolation threshold, more tempering is observed. In figure 5d, we show the average value of $c$ obtained for each city at thresholds above the percolation threshold, which we will refer to as $\bar{c}$. Note that the $\bar{c}$ is observed to be larger and more variable for small cities $\left(A<1,000 \mathrm{~km}^{2}\right)$, decreasing steadily for larger cities (Figure 5d). As a result, larger cities show consistent power-law area distributions even at higher thermal thresholds. Exponential tempering suggests a reduced probability of occurrence of large hot islets for smaller cities and conversely a higher likelihood of encountering them as cities grow even for higher thresholds. Other factors such as urban geometry and disaggregation of heat islets could influence $\bar{c}$ as well but further research will be needed to test that.

\subsection{Islet intensity distribution}

For UHIs, the UHI Intensity is defined as the difference between the mean urban temperature and the mean background temperature of surrounding non-rural regions. An analogous metric for the intra-urban heat islets is defined here as the islet intensity, $\Delta T$, as the difference between the mean temperature of each islet and $T_{t h r}$. This captures the question: How much hotter are the islets than the threshold used to define them? The mean and standard deviation of $\Delta T$ over each islet within a city were found to be equal which, along with the shape of its distribution, were indicative that $\Delta T$ for each city is exponentially distributed, i.e:

$$
p(\Delta T) \propto e^{-\lambda \Delta T}
$$

As a result, we model the islet intensity distribution with a single parameter, $\lambda$ (Figure $6 \mathrm{c}$ and S6). Calculated at the percolation threshold, the values of $\lambda$ across cities display a log-normal distribution with a mean $=2.25 \mathrm{~K}^{-1}$ and s.d. $=1.47 \mathrm{~K}^{-1}$ (Figure 6d; see Dataset $\mathrm{S} 3$ for a complete list). Furthermore, it shows convergence to the mean with 
increasing city size as well (Figure 6e). At a thermal threshold corresponding to the rural background temperature, this corresponds to the conventional metric of mean Urban Heat Island Intensity (Oke, 1982). The scaling observed in the islet size and intensity distributions are analogous to the scaling laws known for areas and mean stages of lakes and wetlands (Bertassello et al., 2018; Cael and Seekell, 2016) and can be used to build the empirical basis for an investigation into the scaling theory of intra-urban heat islets.

\subsection{Convergence in exponents as cities grow}

The area scaling exponent, $\beta$, varies between 1.6 and 2.2 for small cities $\left(a_{c i t y}<1000 \mathrm{~km}^{2}\right)$, but for the larger cities it converges to the mean (see Figure 5c). One explanation for this is statistical, wherein for small cities there are not enough islets obtained at $90 \mathrm{~m}$ resolution which results higher statistical fluctuations about the mean are observed (Figure 8 in Appendix B). As the number of islets increases with city size, steady averaging is achieved that results in convergence towards the mean. However, from an urban growth perspective, this behavior is consistent with several other complex systems that operate within cities (Klinkhamer et al., 2017; Barthelemy, 2016). For smaller cities, the variability reveals the influence of factors unrelated to city size (Cottineau et al., 2017). Land-use and urban infrastructures grow through parallel processes of expansion and densification (Mohajeri et al., 2015). Dense city centers beget more in-fill construction as it becomes a prime spot for economic development. At the same time, sprawling suburbs keep pushing the city boundaries due to the high costs of the inner city. As a result, despite the diversity that smaller cities possess, as the cities grow, they self-organize along a common trajectory (Batty, 2013). Similar convergence is also observed in the islet intensity distribution, $\lambda$ (Figure 6e). On the other hand, the exponential tempering coefficient, $\bar{c}$, converges to 0 (Figure $5 \mathrm{~d}$ ), which means the mega-agglomerations approach a consistent power law even at higher thresholds. This suggests an increase in the proportion of city area that is exposed to higher temperatures (Zhou et al., 2017). This is also in agreement with the observed scaling of aggregated UHI Intensity with the $\log$ of city size (Oke, 1973).

\subsection{Application for assessments}

The narrow distributions of scaling parameters and their convergence are also relevant to the field of urban climate research, for instance, to model the heat exchange between hot areas and their colder surroundings (Oke, 1982). Current numerical weather prediction models, such as Weather Research Forecast (WRF) (Chen et al., 2011), use gridded data formats and, as a result, the perimeter of any heat islet is resolved to the minimum resolution (about $\sim 1-9 \mathrm{~km}^{2}$ ). This results in an under-estimation of urban perimeter boundary which is important for modeling heat exchange across the urban-rural transect. A fractal perimeter of iso-thermal contour lines indicates a larger perimeter of contact with cooler regions, which in turn enables a larger heat flux to dissipate from the heat islets. The inclusion of a correction factor to simulate a rough and convoluted perimeter (with $D \sim 1.33$ ) may improve the modeling of such processes. Furthermore, as the scaling metrics are rather narrowly distributed across diverse cities, we expect such a correction factor to be extendable across all urban areas.

For extreme heat exposure assessment of urban communities, however, analysis of SUHI alone is not enough. Heatstress assessment requires the joint consideration of air temperature and humidity (Oleson et al., 2015). Despite the difference in absolute values of UHI and SUHI, similarities between spatial patterns of the surface and air temperatures have been reported (Schwarz et al., 2012; Henry et al., 1989). Therefore, techniques of scaling based on SUHI patterns can be extended to spatial clusters of UHI as well. The additional challenge is to better understand the superimposition of intra-urban heat islets with the spatial distribution of vulnerable communities (Chapman et al., 2017), such as the poor in mega-cities, the elderly, or critical urban infrastructure such as roads, power grids, and communication networks (Seto et al., 2012; Creutzig et al., 2015). 


\section{Summary}

We show that the spatial structure of Surface Urban Heat Island (SUHI) is strongly fractal for 78 diverse global cities. As a result, it can be conceptualized as a collection of intra-urban heat islets that occur as local heat clusters within the cities. The heat islets have remarkably similar spatial structure as characterized by the fractal dimension $(D)$, as well as a power-law size distribution with exponent, $\beta$ at the percolation threshold. This finding is rather surprising given the diversity of geographic, and socioeconomic constraints in the population of cities studied. At higher thermal thresholds, deviation from power law is observed in the form of an exponential tempering $(c)$, which indicates reduced clustering of extreme heat. Further research into the relationship between urban morphology and exponential tempering can provide some useful insights on urban design solutions for intra-urban heat mitigation.

The selection of a temperature threshold that defines extreme heat varies from region to region depending on their climate. For instance, the National Oceanic and Atmospheric Administration (NOAA) issues a heat stress warning above $33^{\circ} \mathrm{C}$ for some regions in the US, whereas in the tropical regions of India, up to $40^{\circ} \mathrm{C}$ does not warrant a warning (https://www.weather.gov/safety/heat-index). In the absence of a standard definition, use of percentile thermal threshold based on historical records have been recommended (Robinson, 2001). Similarly, instead of setting rural temperature as a benchmark, we present a more flexible characterization of local thermal maxima in the form of islet intensity, $\Delta T$ (Equation 5), from a percentile-based threshold. Furthermore, as the pdf describing their distribution follows an exponential distribution, the intensity parameter $(\lambda)$ can be used to characterize the heterogeneity of thermal extremes and compare across cities. The proposed framework of identifying extreme heat clusters by using incremental thresholds can be used to describe the patterns of extreme heat clusters in any thermal landscape.

While overarching metric such as the ones derived here do not help in answering specific questions pertaining to a particular city, the convergence of the metrics with increasing size does suggest a common attractor for all cities. Both $\lambda$ and $c$ were observed to decrease as the cities grow in size indicating an increased likelihood of occurrence larger and hotter heat islets for mega-cities indicating that their residents are at greater risk of extreme heat stress impacts. This begs the question if this is an inevitable or a desirable trajectory for growing cities? Such questions are of critical importance now, as billions of people add to the urban populations, especially in the developing countries of Africa and Asia. Identifying the common statistical properties of the heat islets across diverse cities provides a means to escape from the geographical malaise of the uniqueness of place, and provides a step towards the improved characterization of the complex urban thermal landscape.

\section{Acknowledgments}

The authors thank the organizers and participants of a series of Complex Networks Synthesis workshops; co-hosted by Purdue University, University of Florida, Helmholtz Centre for Environmental Research, UFZ, and Technical University, Dresden, Germany; which creating a trans-disciplinary collaborative research environment and provided critical input across multiple workshops. A.S. wants to thank the NASA Earth and Space Science Fellowship (Grant number: 80NSSC17K0441) for funding. P.S.C.R. acknowledges the support from NSF Collaborative Research - RIPS Type 2: Resilience Simulation for Water, Power and Road Networks (1441188) and the Lee A. Reith Endowment in the Lyles School of Civil Engineering at Purdue University.

\section{APPENDIX A: Estimation of Land Surface Temperature}

The algorithm used to calculate the Land Surface Temperature is outlined below.

Step 1: TOA radiance

$$
L_{\lambda}=M_{L} \cdot Q_{c a l}+A_{L}
$$


where,

$L_{\lambda}=$ TOA spectral radiance $\left(W / m^{2} * \operatorname{srad} * \mu m\right)$

$M_{L}=$ Band-specific multiplicative rescaling factor from the metadata (RADIANCE_MULT_BAND_x, where $\mathrm{x}$ is the band number)

$A_{L}=$ Band-specific additive rescaling factor from the metadata (RADIANCE_ADD_BAND_x)

$Q_{c a l}=$ Quantized and calibrated standard product pixel values (DN)

Step 2: TOA Brightness Temperature

$$
T=\frac{K_{2}}{\ln \left(\frac{K_{1}}{L_{\lambda}}+1\right)}
$$

where,

$T=$ At-satellite brightness temperature $(\mathrm{K})$

$L_{\lambda}=$ TOA spectral radiance $\left(\mathrm{W} / \mathrm{m}^{2} * \operatorname{srad} * \mu \mathrm{m}\right)$

$K_{1}=$ Band-specific thermal conversion constant from the metadata (K1_CONSTANT_BAND_x)

$K_{2}=$ Band-specific thermal conversion constant from the metadata (K2_CONSTANT_BAND_x)

The band-specific values were obtained from the metadata file. These equations are used for both band 10 and 11, to obtain the temperatures. However, to obtain the actual ground surface temperature, the emissivity needs to be calculated. The codes implemented in R here were derived and modified from ArcGIS toolbox(Walawender et al., 2012).

Step 3: Proportion of vegetation $\left(P_{v}\right)$ and Emmissivity $(e)$ is estimated from NDVI to estimate actual LST:

$$
\begin{gathered}
P_{v}=\frac{N D V I-N D V I_{\min }}{\left(N D V I_{\max }-N D V I_{\min }\right)^{2}} \\
e=0.004 * P_{v}+0.986 \\
L S T=\frac{T}{1+w * \frac{T}{\rho} * \ln (e)}
\end{gathered}
$$

where,

$T=$ At satellite brightness temperature $(\mathrm{K})$ as per equation 9

$w=$ Wavelength of emitted radiation $(11.5 \mu \mathrm{m})$

$\rho=h \times \frac{c}{\sigma}=14380 \mu m K$

$\left(\sigma=\right.$ Boltzmann constant $=1.38 \times 10^{23} \frac{\mathrm{J}}{K}$

$\mathrm{h}=$ Plancks constant $=6.626 \times 10^{34} \mathrm{Js}$

$\mathrm{c}=$ velocity of light $=2.998 \times 10^{8} \frac{\mathrm{m}}{\mathrm{s}}$ )

$e=$ emissivity as per equation 9

\section{Appendix B: Additional Figures}

Figures 7 to 10 are the additional figures attached at the end of this document.

\section{APPENDIX C: Fitting Probability distribution functions}

For fitting probability distributions to the cluster size distribution, a combination of maximum-likelihood fitting methods with goodness-of-fit tests based on the Kolmogorov-Smirnov (KS) statistic and likelihood ratios were used (Clauset et al., 2009). A step-by-step methodology as summarized in Box 1 of (Clauset et al., 2009) and outlined below was followed with the help of R-code provided by Laurent Dubroca and Cosma Shalizi on Clauset's website: http://tuvalu.santafe.edu/ aaronc/powerlaws/. 
1. Estimate the parameters xmin and of the power-law model.

2. Calculate the goodness-of-fit between the data and the power law. If the resulting p-value is greater than 0.1, the power law is a plausible hypothesis for the data, otherwise, it is rejected.

3. Compare the power law with alternative hypotheses via a likelihood ratio test. For each alternative, if the calculated likelihood ratio is significantly different from zero, then its sign indicates whether or not the alternative is favored over the power-law model.

The data were tested for a power-law tail fit and compared against 4 other competing distributions - Exponential, Lognormal, Stretched Exponential (Weibull), and Power-law with an exponential rate of tempering. The basic idea behind the likelihood ratio test is to compute the likelihood of the data under two competing distributions. The one with the higher likelihood is then the better fit. Alternatively, one can calculate the ratio of the two likelihoods, or equivalently the logarithm $\mathrm{R}$ of the ratio, which is positive or negative depending on which distribution is better or zero in the event of a tie. Furthermore, the p-value for the Log-likelihood Ratio is checked and an outcome is selected only if the p-value is less than 0.1 (For a $90 \%$ confidence).

The cluster size distributions for all cities were tested at the percolation temperature, and all of the distributions were found to qualify as a power-law tail (with a p-value of 0.1 , i.e. $90 \%$ confidence). The lower cut-off for power law was found to be under $500 \mathrm{~m}$ for most cities (95\% CI one-sided), this roughly corresponds to the size of an urban block implying that the scaling doesn't extend to the length scales smaller than an urban block. On comparing against the other distribution, we find that 9 of the 78 cities (11.54\%) can also be described as a power-law with exponential tempering: $P(A>a) \propto a^{(-(\beta-1))} e^{(-c \cdot a)}$ with low exponential rates $(c<0.05)$. However, none of them have likelihoods suggesting a Weibull, exponential, or lognormal describe the data better. The table with each city's results is attached as separate excel sheet (Table S2: Tests of fitting exceedance probability distributions).

\section{References}

Allen, M. R., and Coauthors, 2014: Ipcc fifth assessment synthesis report-climate change 2014 synthesis report.

Barthelemy, M., 2016: The structure and dynamics of cities. Cambridge University Press.

Batty, M., 2013: The new science of cities. Mit Press.

Batty, M., and P. A. Longley, 1994: Fractal cities: a geometry of form and function. Academic press.

Bertassello, L. E., P. S. C. Rao, J. W. Jawitz, G. Botter, P. V. Le, P. Kumar, and A. F. Aubeneau, 2018: Wetlandscape fractal topography. Geophysical Research Letters, 45 (14), 6983-6991.

Bettencourt, L., and G. West, 2010: A unified theory of urban living. Nature, 467 (7318), 912.

Buyantuyev, A., and J. Wu, 2010: Urban heat islands and landscape heterogeneity: linking spatiotemporal variations in surface temperatures to land-cover and socioeconomic patterns. Landscape Ecology, 25 (1), 17-33.

Cael, B. B., and D. A. Seekell, 2016: The size-distribution of earths lakes. Scientific Reports, 6, 29633.

Chapman, S., J. E. Watson, A. Salazar, M. Thatcher, and C. A. McAlpine, 2017: The impact of urbanization and climate change on urban temperatures: a systematic review. Landscape Ecology, 32 (10), 1921-1935.

Chen, F., and Coauthors, 2011: The integrated wrf/urban modelling system: development, evaluation, and applications to urban environmental problems. International Journal of Climatology, 31 (2), 273-288.

Chen, Y., 2010: Characterizing growth and form of fractal cities with allometric scaling exponents. Discrete Dynamics in Nature and Society, 2010.

Clauset, A., C. R. Shalizi, and M. E. Newman, 2009: Power-law distributions in empirical data. SIAM Review, 51 (4), 661-703. 
Cottineau, C., E. Hatna, E. Arcaute, and M. Batty, 2017: Diverse cities or the systematic paradox of urban scaling laws. Computers, environment and urban systems, 63, 80-94.

Creutzig, F., G. Baiocchi, R. Bierkandt, P.-P. Pichler, and K. C. Seto, 2015: Global typology of urban energy use and potentials for an urbanization mitigation wedge. Proceedings of the National Academy of Sciences, 112 (20), 6283-6288.

Debbage, N., and J. M. Shepherd, 2015: The urban heat island effect and city contiguity. Computers, Environment and Urban Systems, 54, 181-194.

Fluschnik, T., S. Kriewald, A. García Cantú Ros, B. Zhou, D. E. Reusser, J. P. Kropp, and D. Rybski, 2016: The size distribution, scaling properties and spatial organization of urban clusters: a global and regional percolation perspective. ISPRS International Journal of Geo-Information, 5 (7), 110.

Gangopadhyay, K., and B. Basu, 2009: City size distributions for india and china. Physica A: Statistical Mechanics and its Applications, 388 (13), 2682-2688.

Gonzalez, M. C., C. A. Hidalgo, and A.-L. Barabasi, 2008: Understanding individual human mobility patterns. Nature, 453 (7196), 779.

Gorelick, N., M. Hancher, M. Dixon, S. Ilyushchenko, D. Thau, and R. Moore, 2017: Google earth engine: Planetaryscale geospatial analysis for everyone. Remote Sensing of Environment, 202, 18-27.

Henry, J. A., O. F. Wetterqvist, S. J. Roguski, and S. E. Dicks, 1989: Comparison of satellite, ground-based, and modeling techniques for analyzing the urban heat island. Photogrammetric Engineering and Remote Sensing, 55 (1), $69-76$.

Imhoff, M. L., P. Zhang, R. E. Wolfe, and L. Bounoua, 2010: Remote sensing of the urban heat island effect across biomes in the continental usa. Remote Sensing of Environment, 114 (3), 504-513.

Imre, A. R., and J. Novotnỳ, 2016: Fractals and the korcak-law: a history and a correction. The European Physical Journal H, 41 (1), 69-91.

Isichenko, M., and J. Kalda, 1991: Statistical topography. i. fractal dimension of coastlines and number-area rule for islands. Journal of Nonlinear Science, 1 (3), 255-277.

Isichenko, M. B., 1992: Percolation, statistical topography, and transport in random media. Reviews of Modern Physics, 64 (4), 961.

Klinkhamer, C., E. Krueger, X. Zhan, F. Blumensaat, S. Ukkusuri, and P. S. C. Rao, 2017: Functionally fractal urban networks: Geospatial co-location and homogeneity of infrastructure. arXiv preprint arXiv:1712.03883.

Krueger, E., C. Klinkhamer, C. Urich, X. Zhan, and P. S. C. Rao, 2017: Generic patterns in the evolution of urban water networks: Evidence from a large asian city. Physical Review E, 95 (3), 032312.

Lazzarini, M., P. R. Marpu, and H. Ghedira, 2013: Temperature-land cover interactions: The inversion of urban heat island phenomenon in desert city areas. Remote Sensing of Environment, 130, 136-152.

Li, D., and E. Bou-Zeid, 2013: Synergistic interactions between urban heat islands and heat waves: the impact in cities is larger than the sum of its parts. Journal of Applied Meteorology and Climatology, 52 (9), 2051-2064.

Liu, H., and Q. Weng, 2009: Scaling effect on the relationship between landscape pattern and land surface temperature. Photogrammetric Engineering \& Remote Sensing, 75 (3), 291-304.

Lovejoy, S., and D. Schertzer, 1986: Scale invariance, symmetries, fractals, and stochastic simulations of atmospheric phenomena. Bulletin of the American Meteorological Society, 67 (1), 21-32.

Makse, H. A., J. S. Andrade, M. Batty, S. Havlin, H. E. Stanley, and Coauthors, 1998: Modeling urban growth patterns with correlated percolation. Physical Review E, 58 (6), 7054.

Mandelbrot, B. B., 1975: Stochastic models for the earth's relief, the shape and the fractal dimension of the coastlines, and the number-area rule for islands. Proceedings of the National Academy of Sciences, 72 (10), 3825-3828. 
Meehl, G. A., and C. Tebaldi, 2004: More intense, more frequent, and longer lasting heat waves in the 21 st century. Science, 305 (5686), 994-997.

Mohajeri, N., A. Gudmundsson, and J.-L. Scartezzini, 2015: Expansion and densification of cities: Linking urban form to urban ecology. International Conference on Future Buildings \& Districts Sustainability from Nano to Urban Scale, Lausanne, Switzerland, 9-11.

Newman, M. E., 2005: Power laws, pareto distributions and zipf's law. Contemporary Physics, 46 (5), 323-351.

Oke, T. R., 1973: City size and the urban heat island. Atmospheric Environment (1967), 7 (8), 769-779.

Oke, T. R., 1982: The energetic basis of the urban heat island. Quarterly Journal of the Royal Meteorological Society, 108 (455), 1-24.

Oleson, K., A. Monaghan, O. Wilhelmi, M. Barlage, N. Brunsell, J. Feddema, L. Hu, and D. Steinhoff, 2015: Interactions between urbanization, heat stress, and climate change. Climatic Change, 129 (3-4), 525-541.

Peel, M. C., B. L. Finlayson, and T. A. McMahon, 2007: Updated world map of the köppen-geiger climate classification. Hydrology and Earth System Sciences, 4 (2), 439-473.

Peng, S., and Coauthors, 2011: Surface urban heat island across 419 global big cities. Environmental Science \& Technology, 46 (2), 696-703.

Robinson, P. J., 2001: On the definition of a heat wave. Journal of Applied Meteorology, 40 (4), 762-775.

Rosenzweig, C., W. Solecki, S. A. Hammer, and S. Mehrotra, 2010: Cities lead the way in climate-change action. Nature, 467 (7318), 909.

Rozenfeld, H. D., D. Rybski, J. S. Andrade, M. Batty, H. E. Stanley, and H. A. Makse, 2008: Laws of population growth. Proceedings of the National Academy of Sciences, pnas-0807435 105.

Sahimi, M., and M. Sahimi, 2014: Applications of percolation theory. CRC Press.

Schwarz, N., U. Schlink, U. Franck, and K. Großmann, 2012: Relationship of land surface and air temperatures and its implications for quantifying urban heat island indicatorsan application for the city of leipzig (germany). Ecological Indicators, 18, 693-704.

Seto, K. C., and J. M. Shepherd, 2009: Global urban land-use trends and climate impacts. Current Opinion in Environmental Sustainability, 1 (1), 89-95.

Seto, K. C., and Coauthors, 2012: Urban land teleconnections and sustainability. Proceedings of the National Academy of Sciences, 109 (20), 7687-7692.

Shreevastava, A., P. S. C. Rao, and G. S. McGrath, 2018: Spatial analysis of the surface urban heat island. Land Surface and Cryosphere Remote Sensing IV, International Society for Optics and Photonics, Vol. 10777, 107770C.

Stewart, I. D., and T. R. Oke, 2012: Local climate zones for urban temperature studies. Bulletin of the American Meteorological Society, 93 (12), 1879-1900.

Voogt, J. A., and T. R. Oke, 2003: Thermal remote sensing of urban climates. Remote Sensing of Environment, 86 (3), 370-384.

Walawender, J. P., M. J. Hajto, and P. Iwaniuk, 2012: A new arcgis toolset for automated mapping of land surface temperature with the use of landsat satellite data. Geoscience and Remote Sensing Symposium (IGARSS), 2012 IEEE International, IEEE, 4371-4374.

Weng, Q., 2003: Fractal analysis of satellite-detected urban heat island effect. Photogrammetric Engineering \& Remote Sensing, 69 (5), 555-566.

Yang, S., K. Paik, G. S. McGrath, C. Urich, E. Krueger, P. Kumar, and P. S. C. Rao, 2017: Functional topology of evolving urban drainage networks. Water Resources Research, 53 (11), 8966-8979.

Zhou, B., D. Rybski, and J. P. Kropp, 2013: On the statistics of urban heat island intensity. Geophysical Research Letters, 40 (20), 5486-5491. 
Zhou, B., D. Rybski, and J. P. Kropp, 2017: The role of city size and urban form in the surface urban heat island. Scientific Reports, 7 (1), 4791.

Zhou, W., G. Huang, and M. L. Cadenasso, 2011: Does spatial configuration matter? understanding the effects of land cover pattern on land surface temperature in urban landscapes. Landscape and Urban Planning, 102 (1), 54-63. 


\section{Figures}

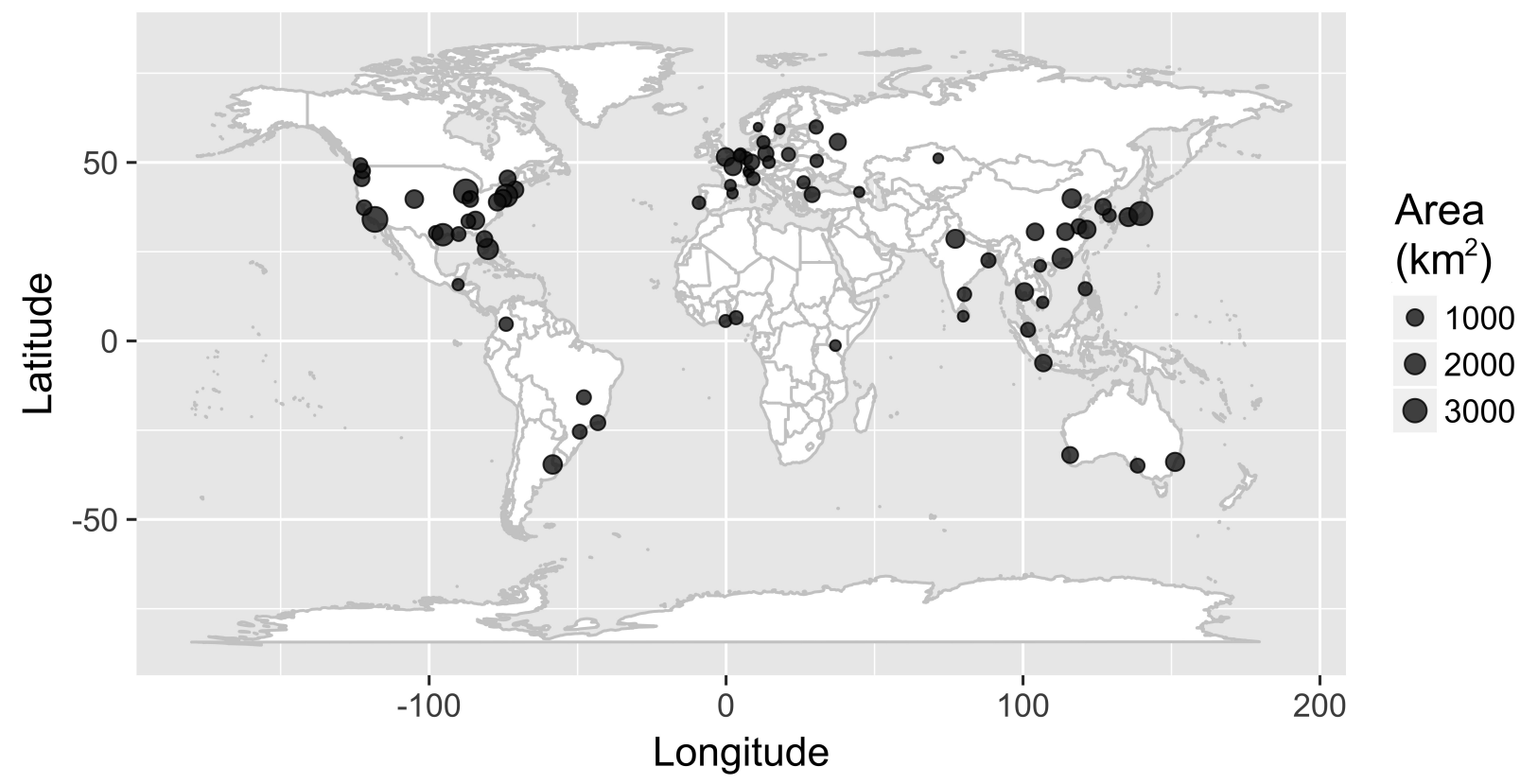

Figure 1: Map of the selected 78 cities chosen for this study. The size of marker in an indicator of the area of cities measured using the Urban land use class of MODIS Land Cover Type dataset. 

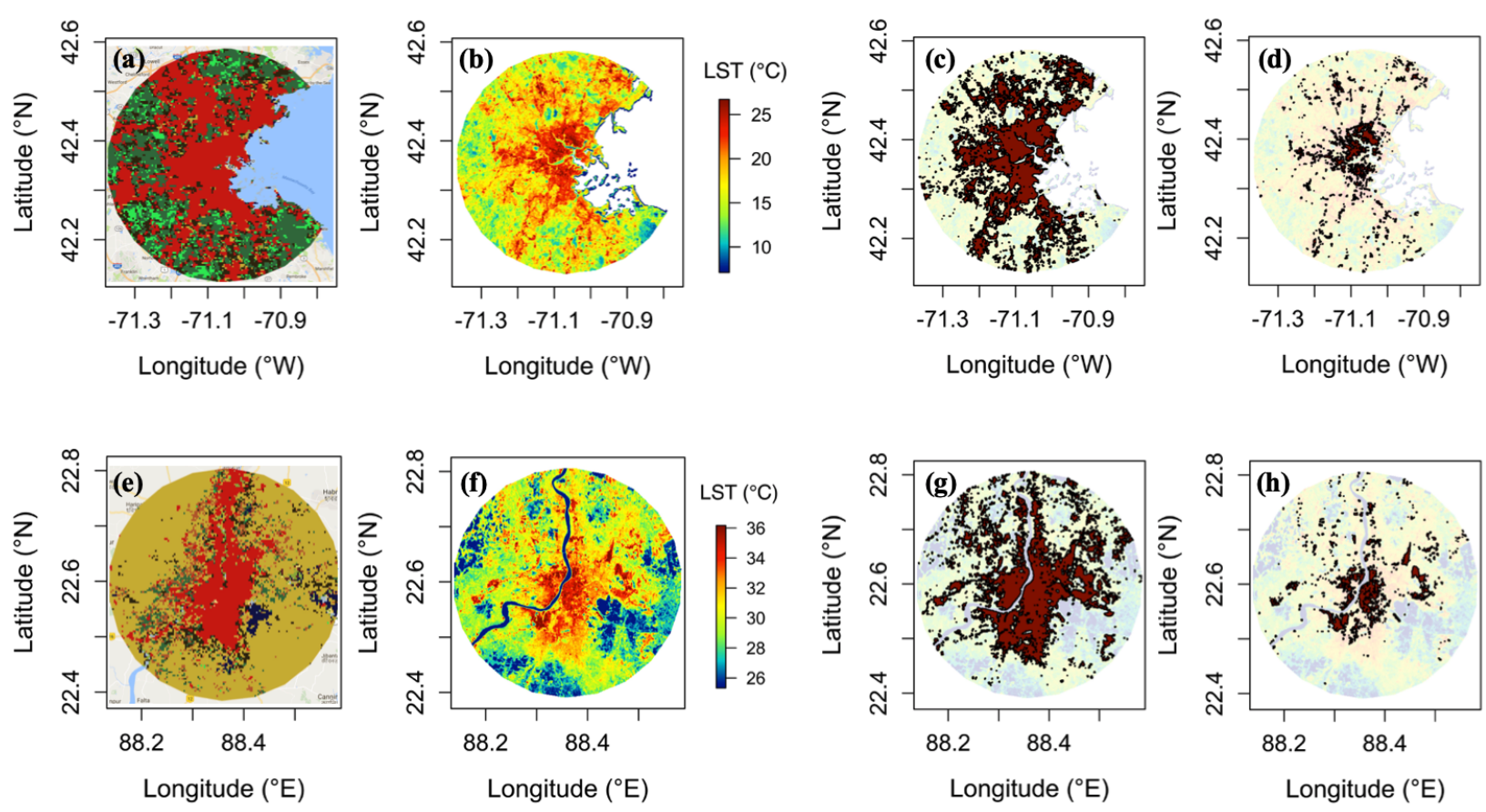

Figure 2: Maps for Boston (top) and Kolkata (bottom) are shown here as examples. (a, e) Land use map derived from MODIS - Land Cover Type dataset for the year 2016. (b, f) Land Surface Temperature (in ${ }^{\circ} \mathrm{C}$ ) map derived from Landsat 8. (c, g) Clusters of high heat (Islets) above the statistical mode of temperatures, i.e. the most frequently encountered temperature $\left(19^{\circ} \mathrm{C}\right.$ for Boston and $32^{\circ} \mathrm{C}$ for Kolkata) obtained using Moore neighborhood clustering algorithm are indicated as red. $(\mathrm{d}, \mathrm{h})$ Extreme high heat islets obtained at the 95th percentile temperature of each city. Note the irregularity in the islets' perimeters and the disparity in their sizes.
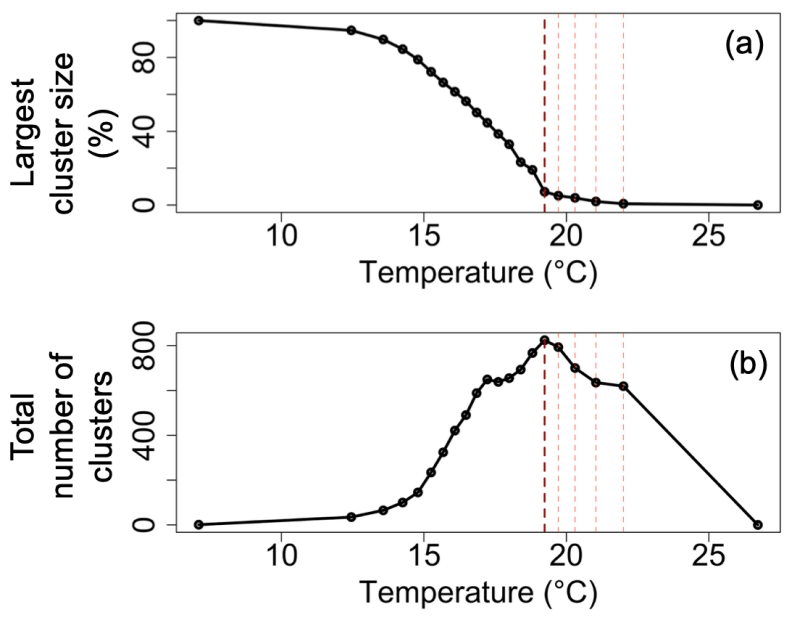

Figure 3: (a) Plot of largest cluster size as a function of thermal threshold for the case of Boston city (b) Total number of clusters shown for each thermal threshold. The first dashed red line shows the percolation threshold $\left(75^{\text {th }}\right.$ percentile in this case) identified as the threshold where the total number of clusters is the maximum and below which the largest connected component emerges. Lighter red lines towards its right mark the subsequent percentiles of threshold which were considered for the analysis. 

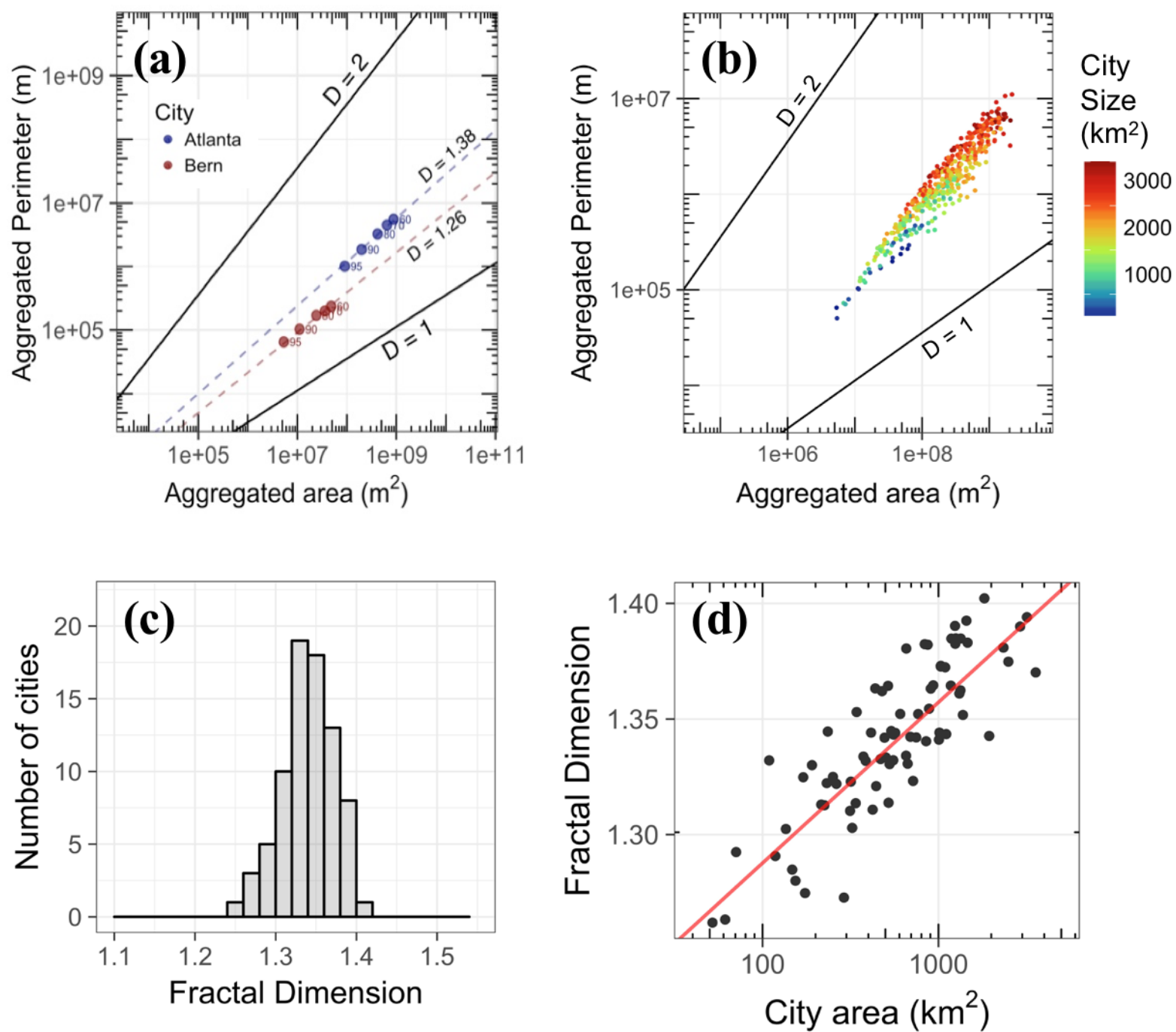

Figure 4: (a) Aggregated perimeters versus aggregated areas at 60, 70, 80, and 90 percentiles thresholds are shown here for two cities, Bern (in red) and Atlanta (in blue), demonstrating the same ratio of $\log ($ Area) and $\log$ (Perimeter) and hence the same Fractal Dimension (D) of iso-thermal contour lines as indicated by the grey, dashed lines show examples of two cities with $D=1.38$ for Atlanta and $D=1.26$ for Bern. D of the perimeter of a circle $(D=1)$ and a space-filling plane $(D=2)$ are plotted to show the physical bounds for $\mathrm{D}$. (b) The same plot for all cities shown with a single colour attributed to each city that corresponds to its area. (c) Histogram of $D$ for all cities at their respective percolation thresholds with mean $=1.33 \pm 0.007(95 \% \mathrm{CI})$. (d) D as a function of the city area. This plot serves to illustrate that $D$ increases with city area as per $D=0.0695 \log A_{\text {city }}+1.15\left(R^{2}=0.7\right)$. 

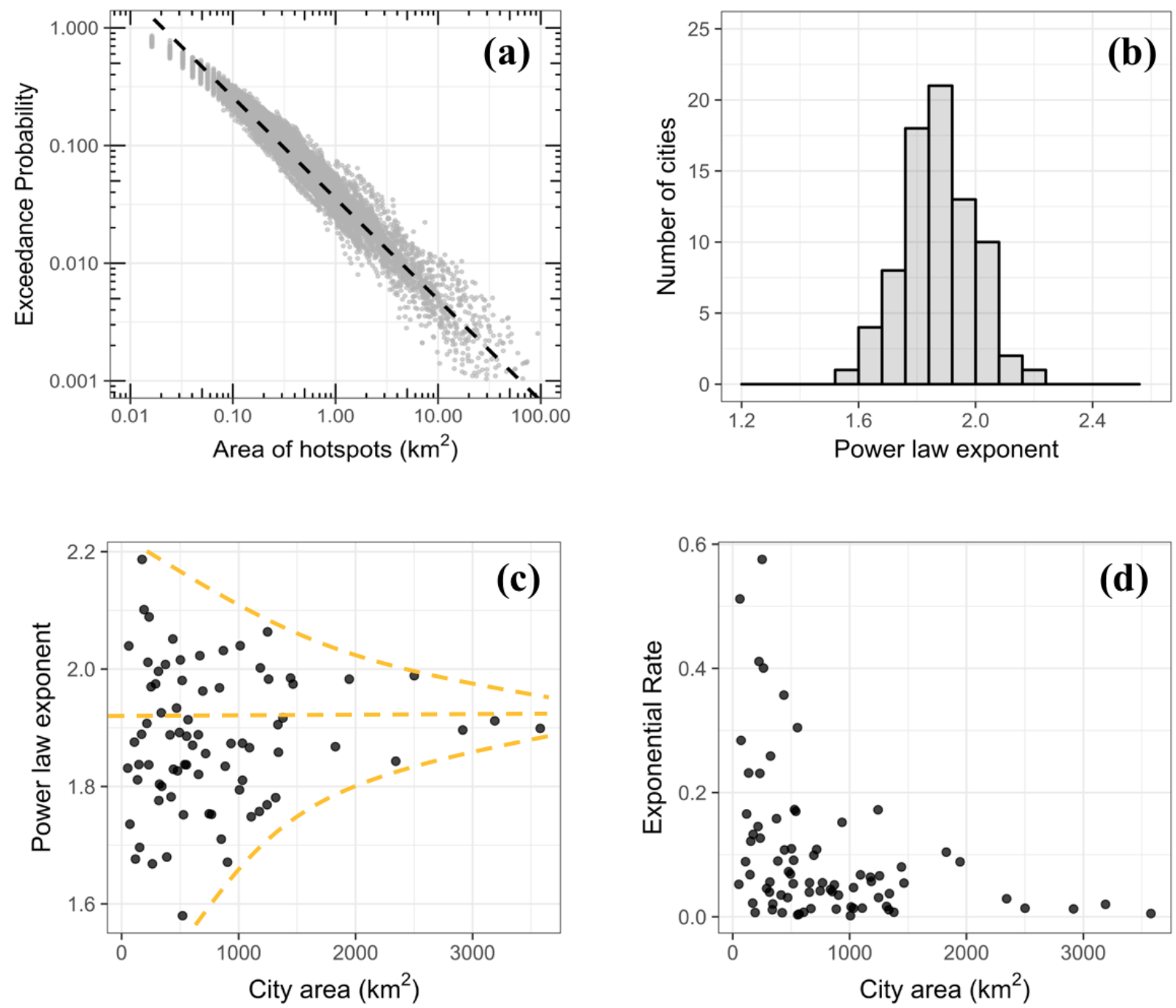

Figure 5: (a) Area Exceedance Probability Distributions for all cities at their respective percolation threshold are shown here in grey. Overlaid as a dashed black line is the line demonstrating the mean scaling exponent, $\beta=1.88$. (b) A histogram of $\beta$ of all cities. (c) Scatter plot of $\beta$ and city area for each city. Yellow dashed lines serve to highlight this convergence of $\beta$ to mean with an increase in the city area. (d) Scatter plot of mean exponential tempering coefficient, $\bar{c}$, calculated as an average of tempering coefficients $(c)$ obtained at temperatures above the percolation threshold. It is shown to rapidly decreasing to $\bar{c}=0$ with increasing city area. Each black dot represents a single city. 

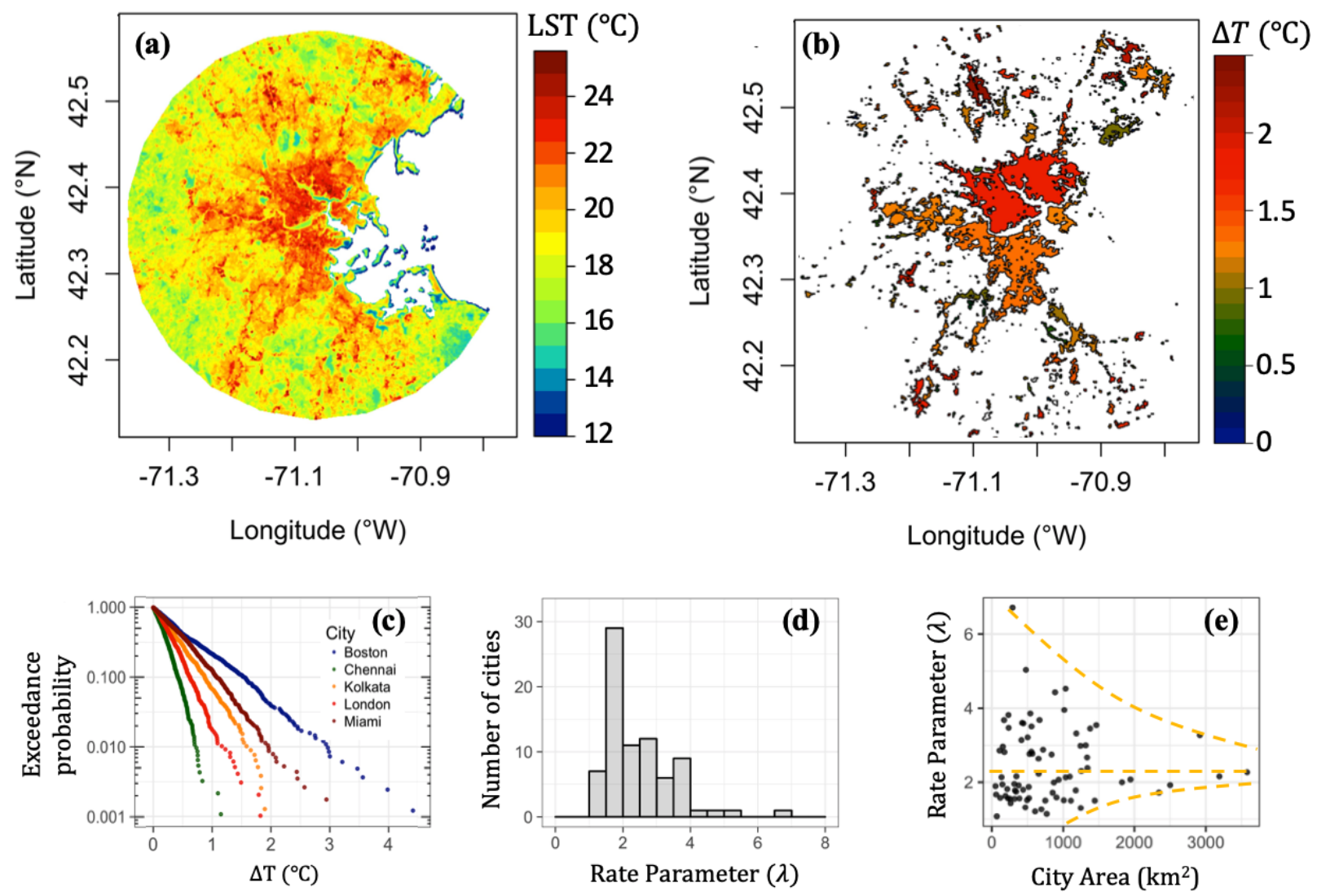

Figure 6: (a) Land Surface Temperature map of Boston (b) Map of heat islets obtained at mode temperature $\left(19^{\circ} \mathrm{C}\right.$, in this case) with colour representing the islet intensity $(\Delta T)$ above the mode. (c) Examples of empirical pdf of $\Delta T$ for 5 selected cities shown on a semi-log graph at their respective mode temperatures to illustrate the disparity in exponential pdfs of $\Delta T$. Similar plot with all cities can be found as Figure S6. (d) Histogram of rate parameter $\lambda$ (Eqn. 5) with mean $=2.25 K^{-1}$. (e) Scatter plot of $\lambda$ and area of all cities. Yellow dashed lines show the converging behaviour of $\lambda$ with increasing area. 
(a) $\mathrm{p}=10$

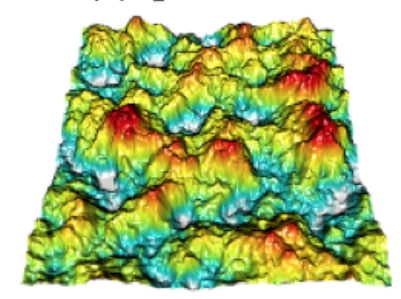

(d) $p=40$

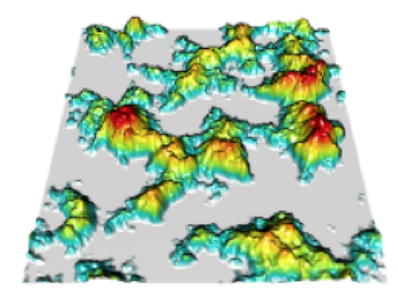

(g) $p=70$

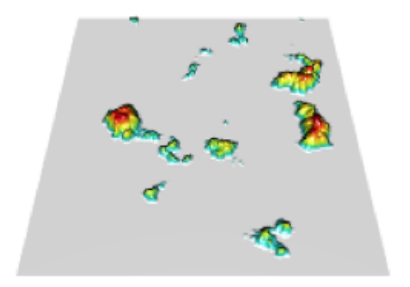

(b) $p=20$

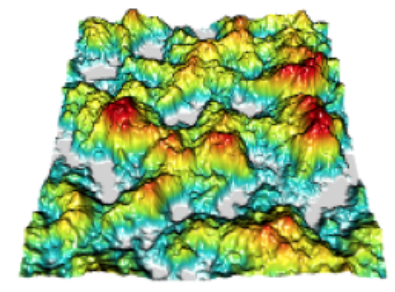

(c) $p=30$

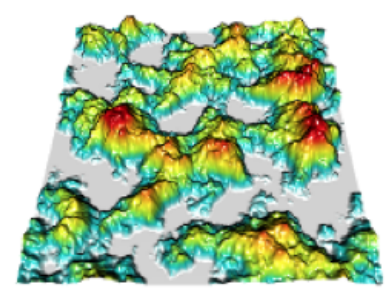

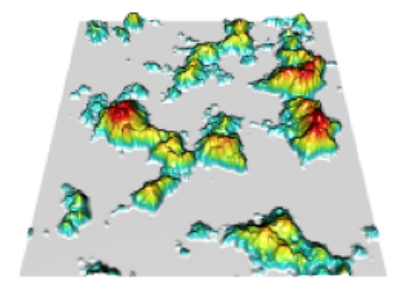

(f) $\mathrm{p}=60$

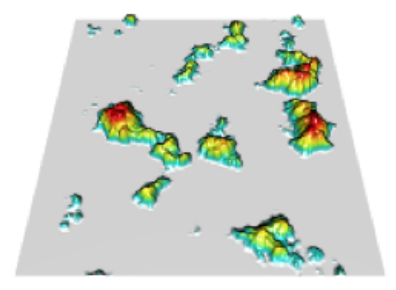

(i) $\mathrm{p}=90$

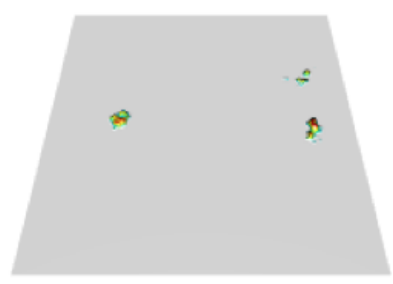

Figure 7: Illustrated above in an example of thresholding by percentile. The thermal maps are represented as 3-d elevation maps where height, as well as color, corresponds to a higher temperature. For each percentile of the thermal threshold, the areas above that are selected, and connected pixels (by Moore neighborhood) are grouped into a cluster. Figures (a-i) show the clusters that emerge above 9 incremental percentiles (shown as $\mathrm{p}$, here). 


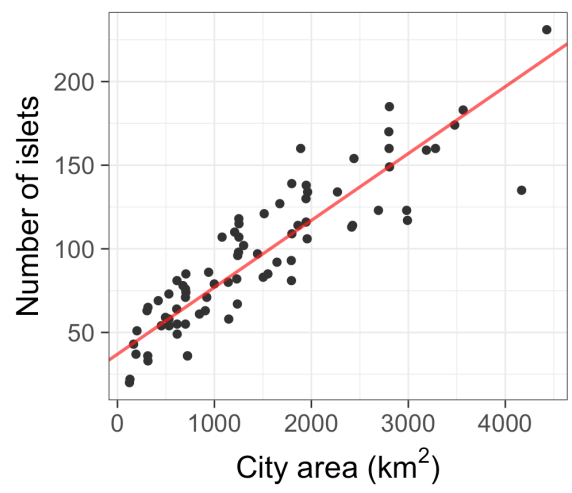

Figure 8: Scatter plot showing the correlation between number of islets and city size that scales linearly as $N=$ $0.038 * A_{\text {city }}+40\left(R^{2}=0.8\right)$ as indicated by the red line.

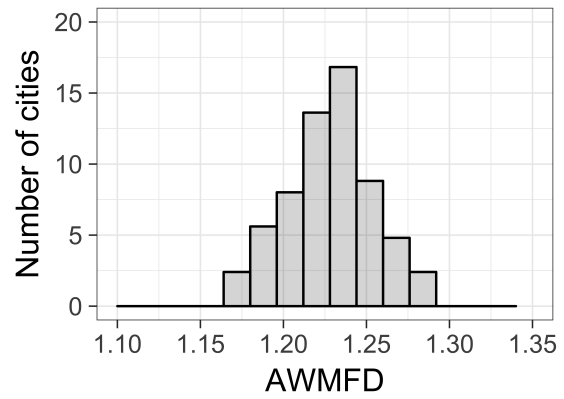

Figure 9: Histogram of Area Weighted Mean Fractal Dimension (AWMFD) for 78 cities.

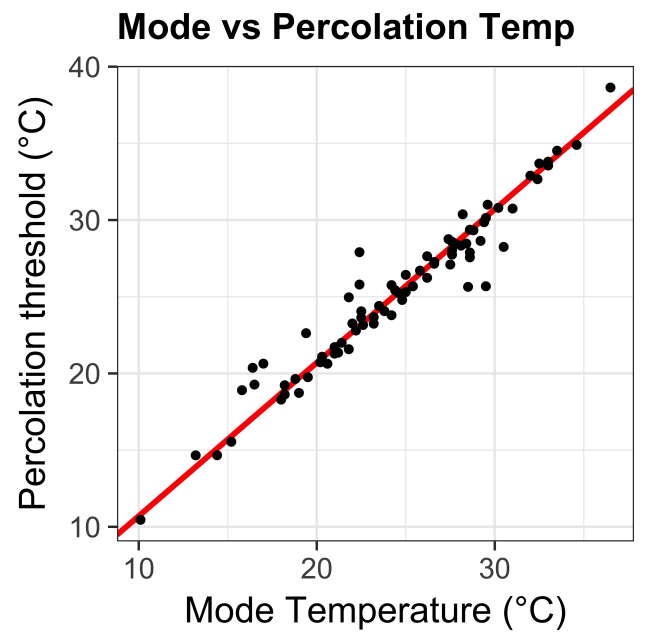

Figure 10: Scatter plot showing the correlation between mode temperature and the percolation threshold $\left(R^{2}=0.93\right)$ 